\title{
Reliability of a Radio Environment Map: Case of Spatial Interpolation Techniques
}

\author{
D. Denkovski, V. Atanasovski, L. Gavrilovska \\ Faculty of Electrical Engineering and Information \\ Technologies, Ss Cyril and Methodius University in Skopje \\ Skopje, Macedonia \\ Email: \{danield, vladimir, liljana $\} @$ feit.ukim.edu.mk
}

\author{
J. Riihijärvi, P. Mähönen \\ Institute for Networked Systems \\ RWTH Aachen University \\ Aachen, Germany \\ Email: \{jar, pma $\} @$ inets.rwth-aachen.de
}

\begin{abstract}
Recent advances in radio environmental mapping enable novel, practical and efficient cognitive radio and dynamic spectrum access solutions. A crucial aspect of such solutions is to ensure the reliability of the constructed Radio Environmental Maps (REMs). Especially important is the accurate and up-todate Radio Interference Field (RIF) estimation based on distributed spectrum use measurements. This paper analyzes the use of spatial interpolation techniques that allow robust, yet sufficiently reliable, RIF estimation from a limited number of field measurements. Several spatial interpolation techniques based on Inverse Distance Weighting (IDW) are analyzed and compared in terms of reliability bounds of the interpolation errors for an indoor environment. Performance evaluation using REM prototype implementation and a wireless testbed shows that the spatial interpolation techniques can provide a robust and reliable RIF estimation within the entire REM concept.
\end{abstract}

Keywords-Radio Environmental Map (REM), Radio Interference Field (RIF), spatial interpolation, IDW, reliability.

\section{INTRODUCTION}

One of the key concepts of Mitola's original definition of cognitive radio (CR) [1] is the use of context information for adaptation and performance optimization. While much of the recent work in the cognitive radio domain has focused on dynamic spectrum access (DSA) related problems, this more general goal remains highly topical. In fact, several recent research results have started to demonstrate the potential performance gains that can be obtained by incorporating information on the state of the radio environment into radio resource management decisions. The concept of Radio Environment Maps (REMs) [2],[3] has become very prominent in this line of work. REMs can be thought of as databases or knowledge bases in which different kinds of radio environmental information can be stored. Examples of relevant information types include locations of transmitters and receivers, models of the propagation environment, and various spectrum use measurements conducted by wireless devices. Based on such information further details of the radio environment can be estimated and modeled, such as levels of interference a particular transmission would cause in its surroundings. Despite the potential benefits, most of the work on REMs carried out until has been theoretical in nature, with less focus on practical use cases and implementation details.
In this paper we discuss our work towards implementing a flexible REM prototype, and in particular on our approaches for robust estimation of interference and coverage characteristics of wireless systems based on small number of measurements. When a large amount of data is available, wellknown techniques from spatial statistics can be applied for accurate estimation as discussed in [4],[5]. However, many of these techniques do not work well if only small amount of measurements are available. We show how robust estimates can be made also in such situations, significantly improving the practical applicability of REM designs for network optimization especially in the start up phase of the system. We also discuss our overall REM design and implementation in some level of detail, especially focusing on the functional architecture, the data model adopted for the REM knowledge base, and how these are mapped to the actual implementation. We emphasize that even though we focus here on one particular use case, namely on coverage estimation, the architecture and implementation of the REM are quite general, and can be used in a much broader range of applications.

The rest of this paper is structured as follows. Section II explains in details the minimum required REM backend functional blocks and interfaces for practical deployment. Section III provides the explanation on three different inverse distance weighting (IDW) spatial interpolation techniques, while Section IV evaluates the REM reliability using the explained techniques. Finally, section V concludes the paper.

\section{REM DESIGN AND PROTOTYPE IMPLEMENTATION}

REMs are a fundamental enabling technology to implement practical cognitive radio networks and dynamic spectrum access solutions. This section presents a general REM architecture and prototype, the REM data model and representation with special focus on the radio interference field (RIF) estimation through spatial interpolation.

\section{A. REM Architecture}

A general architecture for REM generation and evaluation should comprise several key functionalities, starting from the spectrum measurements execution and data acquisition, through the data processing and REM construction, to the REM data presentation and utilization for various spectrum 
management purposes. In order to satisfy these requirements, a functional REM architecture (Fig. 1) should consist of (at least) the following functional entities [6]: Measurement Capable Devices, REM data Storage and Acquisition unit, REM Manager and a REM User. The subsequent paragraphs briefly explain these entities and the respective interfaces.

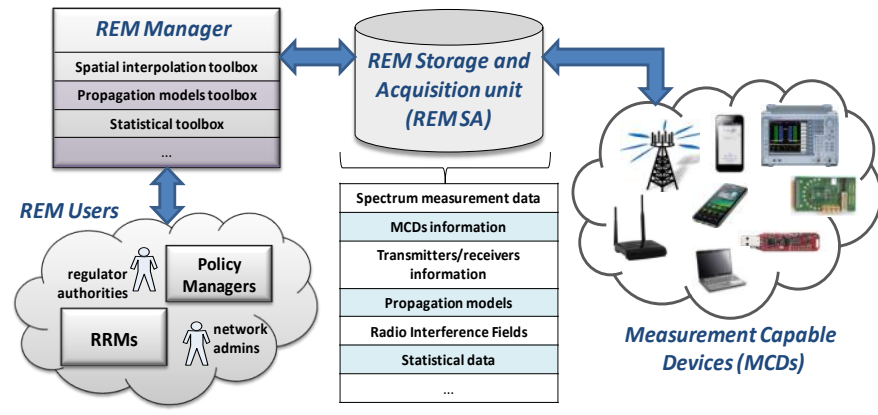

Figure 1. The developed functional REM architecture.

Measurement Capable Devices (MCDs). The REM architecture should be able to integrate different types of spectrum sensing capable devices, considering their limitations, taking advantage of the diversity. The MCDs report their measurements to the REM storage (which can be implemented centrally or in distributed manner) and provide options for remote reconfiguration and measurements querying. The REM architecture envisions and allows for the usage of the measurement capabilities of existing infrastructure components such as base stations, terminals etc., or a dedicated sensor network. These different types of MCDs should be seamlessly integrated into the REM architecture, i.e. the referred should support heterogeneous MCD deployments.

REM data Storage and Acquisition unit (REM SA) represents the main data storage in the REM architecture. Besides the spectrum measurement data coming from the different types of MCDs, the REM SA should keep REMrelevant information, such as information about the positions and configuration on radio transmitters and receivers, environment characteristics, as well as restituted REM processed data. Section II. B. provides more information on the constructed REM data model.

REM Manager is a fundamental part of REM architecture performing the main processing tasks for REM data creation and evaluation. It should be modularly constituted, comprising various toolboxes that serve for localization of transmitters, statistical analyses of the spectrum usage, assessment of the environment propagation characteristics, estimation of RIFs etc. Particularly, the spatial interpolation toolbox (the focus of this paper) should provide the ability to synthesize RIFs using the limited amount of spectrum data stored in the REM SA.

REM users. The REM data can be utilized by different types of REM users, i.e. entities that govern the frequency/power allocation, spectrum access/(re-)usage, network optimization etc. Representative examples are radio resource and policy managers, network administrators and regulator entities.

The REM architecture (Fig. 1) comprises three main interfaces, i.e. a MCD-REM SA interface handling the measurement data reporting and the MCDs registration and (re)configuration, a REM SA-REM Manager interface relaying the communication between the referred entities in terms of the extraction of measurement data and restitution of processed REM data; and a REM Manager-REM User interface providing the REM Users access to REM data and ability to query on-demand some specific REM processing.

\section{B. Types of REM Information}

The current REM data model [7] consists of three major categories of information:

1. Location and configuration information of transmitters, receivers and possible dedicated spectrum sensors. This information can either be preconfigured (for example, obtained from the operator deployment records) or inferred using, for example, localization and transmit power estimation techniques.

2. Information about the environmental characteristics, such as statistical propagation models.

3. Radio interference fields, which consist of spatial "maps" of received signal strengths, and other measurable quantities defined over a given region.

Note that these information types are not independent of each other. For example, given transmitter locations and configurations together with a propagation model, the resulting RIF can be estimated. On the other hand, if nothing is known about the transmitters, the MCDs can gather information about the RIF corresponding to power measurements on a particular channel, and these measurements can be used to infer the transmitter configuration in the region. Due to space reasons we focus here on one type of estimation problem related to RIF estimation, namely the spatial interpolation or spatial estimation of the RIF structure from a limited number of measurements towards regions in which measurements are not directly available.

Such a spatial interpolation problem is very important in a number of application scenarios. For example, a wireless network performing dynamic spectrum access can use interpolation techniques for estimating the coverage area of the primary system, and take that estimate into account when deciding on the used transmit power. Cellular network operators can exploit these methods for interpreting results from drive tests, or in the future for processing data from measurements carried out by mobile devices in the fashion of the ongoing Minimization of Drive Tests work in 3GPP [8]. The propagation and RIF maps thus obtained have in turn several applications for network operators, such assisting in network planning, fault detection, and so on [3]. Finally, regulators and dedicated public bodies could use these techniques for large-scale estimation of how radio frequencies are being actually used, and thereby either track compliance to regulations, or estimate the effectiveness of frequency planning carried out by them.

\section{REM Spatial Interpolation Toolbox}

This subsection focuses on the aspects of spatial interpolation which is tightly related to the RIF estimation feature of the REM Manager. The subsequent classification of 
the existing spatial interpolation methods intends to briefly explore the possibilities for the RIF's estimation problem.

There are a variety of interpolation methods which target the spatial interpolation [9] problem in different scientific and research areas. In general, different interpolation methods can be divided in three main groups, i.e. local neighborhood approaches, geostatistical approaches and variational interpolation approaches, each explained below.

Interpolation based on local neighborhood: These methods assume that the influence of a spatial point is limited with the distance. The interpolated values are computed by predefined functions that reflect the neighboring points influence in the most realistic manner. The most commonly used methods and their variations in the literature are Inverse Distance Weighted interpolation [10],[11], Natural Neighbor interpolation [12] and Triangular Irregular Network (TIN) interpolation [13].

Geostatistical interpolation: This type of interpolation extends far beyond the basic interpolation problems and relies on statistical models that are based on the theory of random functions and variables to model the uncertainty associated with the spatial estimation process. The most widely used geostatical interpolation is based on the kriging method and its variations [9]. These are essentially optimal linear interpolation techniques in the sense of having minimum rootmean-squared-error. However, in order to apply kriging, the correlation structure of the data has to be either known, or estimated from the data. This is difficult to do reliably if only small number of measurements is available, and can lead into unreliable results and lack of robustness.

Variational interpolation: The variational interpolation approach is based on the assumption that the interpolation function should have very small deviations from the data points while tending to be as smooth as possible. These two requirements are combined into a single condition that represents a spline function that reflects the interpolation method. The Thin Plate Spline (TPS) interpolations [14] are the most widely used variational interpolation methods.

This paper focuses particularly on the IDW based spatial interpolation methods and variations as the most applicable RIF estimation methods, due to their robustness and lack of need for second-order structure knowledge. In addition, these methods provide the flexibility to cope with different sensor geometries and limited number of spatial measurements.

\section{REM prototype implementation}

The REM architecture comprising the previously detailed components and interfaces, complying with the REM data model, information flows and processing, has been realized into a REM prototype [7],[15],[16]. It was developed as $\mathrm{C}++/ \mathrm{C \#}$ based modular structure, including an SQL database based realization of the REM storage with the defined data model. The REM prototype incorporates support for several heterogeneous MCDs, such as high-precision spectrum analyzers (Anritsu MS2690A, R\&S FSL6), commercially available mid- and low-end spectrum sensors (USRP2, TI eZ430 RF2500, SunSPOT) and a custom made versatile spectrum sensing device (IMEC's SCALable raDIO [17]). The REM Manager implementation includes a spatial interpolation toolbox (performing IDW based interpolation), a statisticalanalyses toolbox (estimating propagation models, empirical probability models, historical spectrum occupancy etc.) and a transmitter localization toolbox (performing an ML estimation of transmitter location and transmit power [18]).

\section{SPATIAL INTERPOLATION BASED ON INVERSE DiSTANCE WEIGHTING METHODS}

The IDW methods are the simplest and the most robust spatial interpolation methods [10],[11]. They represent a class of local neighborhood methods that assume that each spatial point influences the spatial field only up to a certain distance by the means of using inverse distance weighting coefficients in the interpolation function. The widespread usage of these interpolation methods is owed to their flexibility, i.e. ability to operate in different scenarios with regularly-placed or irregularly-scattered spatial data. Furthermore, they have the capability to robustly work with limited number of samples, whereas geostatistical based approaches require significant sample size for statistical correctness.

In the following we elaborate on three IDW interpolation methods: the classical IDW method [10], a modified version of the classical IDW method [10] and a modified Shepard's method [11], each explained in the next subsections. Although the presented interpolation functions work on two-dimensional data, only slight modifications are required for the $3 \mathrm{D}$ case.

\section{A. Classic IDW Method}

The classical IDW method [10] uses all the available spatial data observations $(N)$ to perform the interpolation:

$$
f(x, y)=\sum_{i=1}^{N} d_{i}^{-d_{\exp }} P_{i} / \sum_{i=1}^{N} d_{i}^{-d_{\exp }} .
$$

Here, $P_{i}$ is the measured output at the point $\left(x_{i}, y_{i}\right), d_{i}$ is the Euclidean distance between the referred point and the interpolation point $(x, y)$ and $d_{\exp }$ is the distance exponent.

There are several drawbacks of this classical IDW method. The processing requirements rise as the number of observed spatial points increases and the method does not take the direction into account. Finally, this method assigns a zero gradient of the interpolation surface at the observed locations resulting in each of them representing an extreme.

\section{B. Modified classic IDW method}

The modified version [10] of the classic IDW method accounts for the direction, the number and set of considered neighboring points and the slope of the interpolation function. The estimated value is calculated with the subsequent formula:

$$
f(x, y)=\sum_{d_{i} \leq r} w_{i}\left(P_{i}+\Delta P_{i}\right) / \sum_{d_{i} \leq r} w_{i} .
$$

Here, only the spatial points in a circle of radius $r$ around the interpolation point are taken into account. In addition, the number of considered observed spatial data points is limited to be in the range $\left[N_{\min }, N_{\max }\right.$ ], thus having an adaptive radius when the limits are exceeded. The weighting coefficients for each neighboring point are calculated as in

$$
w_{i}=\left(s_{i}\right)^{d_{\exp }}\left(1+t_{i}\right) \text {, }
$$


where $s_{i}$ represents the inverse distance coefficient and $t_{i}$ is accounting for the directivity of the interpolation points. It can take values in the range $[0,2]$, and the limits correspond to the cases of neighboring points in the same or the opposite direction respective to the interpolation point. Finally, $\Delta P_{i}$ relates to the slope of the interpolation function calculated as

$$
\Delta P_{i}=\left[A_{i}\left(x-x_{i}\right)+B_{i}\left(y-y_{i}\right)\right]\left[\frac{v}{v+d_{i}}\right],
$$

where $A_{i}$ and $B_{i}$ represent the desired slopes in the $x$ and $y$ directions at the data point $\left(x_{i}, y_{i}\right)$, and $v$ is the parameter bounding the maximums slope effect.

\section{Modified Shepard's Method}

The IDW based modified Shepard's method (MSM) [11] is a local interpolation that makes the estimation based on multivariate functions, often referred as nodal functions. This is the main enhancement compared to the classical IDW methods. Namely, this method fits a local real multivariate function (two-dimensional surface) for each observed spatial point, obtained with weighted least squares (WLS) fitting on the nearest neighbors values. The nodal functions can take different analytical forms (may be quadratic, linear, etc.), and the appropriate one should be selected in compliance with the inspected phenomena. The estimation of their parameters for an observed location $\left(x_{k}, y_{k}\right)$ is performed minimizing

$$
\sum_{i=1}^{N} w_{i}\left(x_{k}, y_{k}\right)\left[Q_{k}\left(x_{i}, y_{i}\right)-P_{i}\right]^{2},
$$

where $Q_{k}\left(x_{i}, y_{i}\right)$ is the output of the nodal function of the data point $\left(x_{k}, y_{k}\right)$ at the location of the neighboring point $\left(x_{i}, y_{i}\right)$. The weighting coefficient $w_{i}(x, y)$ assigned to the point $i$ at an arbitrary location $(x, y)$, is calculated as

$$
w_{i}(x, y)=\left\{\begin{array}{cl}
{\left[\left(R_{q}-d_{i}\right) / R_{q} d_{i}\right]^{d_{\exp }}} & d_{i} \leq R_{q} \\
0 & d_{i}>R_{q}
\end{array} .\right.
$$

In this formula, $R_{q}$ represents the radius of influence about node $\left(x_{i}, y_{i}\right)$. Finally, the interpolation value for an arbitrary spatial point $(x, y)$ is calculated using

$$
f(x, y)=\sum_{k=1}^{N} W_{k}(x, y) Q_{k}(x, y) / \sum_{k=1}^{N} W_{k}(x, y),
$$

where $Q_{k}$ and $W_{k}$ are the output of the nodal function of the data point $k$, and the weight assigned to the referred neighboring point at location $(x, y)$. The weights $W_{k}$ are once again calculated using the same formula as in (6), replacing $R_{q}$ with $R_{w}$. Here, $R_{w}$ represents the maximum distance at which a neighboring point (its nodal function output) is taken into account. Instead of using fixed radii of influence $R_{q}$ and $R_{w}$, another approach can be to fix the number of considered neighboring points $N_{q}$ and $N_{w}$, adapting the radii accordingly.

\section{General Remarks on IDW Based Methods}

The IDW based methods have been widely used for spatial interpolation of various observations. Each phenomenon has a different spatio-temporal distribution yielding different estimation performances. In most of the cases, a distance exponent $d_{\text {exp }}$ equal to two results in best performances.
When the radio propagation environment is in the focus of investigation, the IDW methods require particular tuning to adapt to the nature of this phenomenon. The MSM interpolation is the most flexible of the three considered. A reasonable solution for the RIF interpolation is to have an adaptive $N_{q}$ and $N_{w}$ parameters (depending on the interpolated location), providing the ability of the interpolation function to adjust to the specific surrounding (obstacles, walls etc.).

The analysis in section IV will focus on the aspects of using and proper tuning of these IDW spatial interpolation methods for the RIF estimation.

\section{PERFormance EVAluation OF IDW BASED SPATIAL INTERPOLATION TECHNIQUES}

This section focuses on the performance evaluation of the IDW based spatial interpolations, when applied to the RIF estimation problem. The aim is to test the behavior of the methods detailed in Section III in terms of the RIF estimation reliability with a limited number of MCDs. For these purposes, a testbed comprising ten TI eZ430 RF2500 devices and three USRP2 devices was set up in an indoor environment. The devices were placed in a $25 \mathrm{~m}^{2}$ area in a classroom having a large number of chairs and tables as obstacles and shadowers. The TI sensors were used as MCDs, while the USRP2 devices were employed as signal sources generating $5 \mathrm{MHz}$ wide OFDM signals. The experiments focused on the $2.4 \mathrm{GHz}$ ISM band evaluation. Fig. 2 illustrates the placement of the sensors and the signal sources. A total of 24 different scenarios (combinations) were tested, having each of the signal sources active as a single transmitter or in a pair of transmitters, with three possible transmit power levels, i.e. $-5,-15$ and $-25 \mathrm{dBm}$. Each inspected scenario was evaluated for 5 minutes to gain sufficient time domain statistics. It is important to note that the radio environment during the measurements was not completely controlled, and some of the errors may originate from the outer interference.

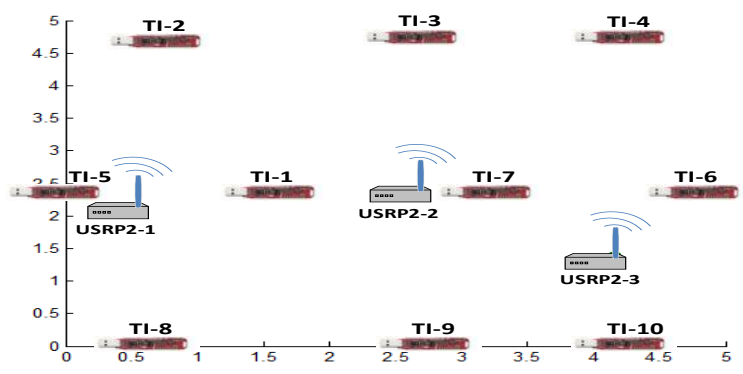

Figure 2. Evaluation scenario.

The IDW methods of interest (Section III) are: the classical IDW, the modified classical IDW, the modified Shepard's method, referred as IDW, IDWM and MSM, respectively. An adaptive MSM (AMSM) approach, which used adaptive $N_{q}$ and $N_{w}$ parameters depending on the location of the interpolation point was also tested. Oppositely, the classical MSM interpolation used static parameters, proven to give the best results in overall for all interpolation points. An additional modification of the MSM approach was also evaluated. Namely, this approach used an inverse covariance matrix of 
the input values ( $x, y$ coordinates) for the WLS estimation of the nodal functions parameters, instead of using the proposed weights in (6). Respectively, the MSM and AMSM methods taking this approach are subsequently referred as MSMC and AMSMC. All MSM approaches used linear nodal functions proven to offer better results for small number of observations.

The evaluation on each of the abovementioned methods focused on the error, the absolute error (AE) and the mean absolute error (MAE) per sensor. The calculations of the errors were made with a sensors exclusion approach, averaging over all combinations starting from nine (out of ten) active sensors down to only five active ones. The errors were calculated as residuals of the interpolated values and the values measured by the excluded sensors. Only the corner sensors with IDs 2, 4, 8 and 10 were not excluded to alleviate the border effects.

The obtained results are cumulative for all 24 inspected scenarios. They are presented as box plots, where the central mark is the median, the edges of the box are the $25^{\text {th }}$ and $75^{\text {th }}$ percentiles and the whiskers extend to the most extreme data points not considered as "outliers". The used whisker length is 1.5 times the interquartile range.

Fig. 3 presents the comparison between the different IDW methods in terms of the MAE per sensor for the case of nine active sensors. In addition, the figure presents the impact of the distance exponent in the interpolation MAE. The results prove that the IDW methods generally perform better when the distance exponent $d_{\exp }$ is equal to 1. The AMSMC method with $d_{\exp }=1$ is the most reliable interpolation in overall, because it offers the lowest and the less variable MAE. It is important to note that the MSM based methods do not perform well when the used $d_{\exp }$ is equal to 2 . The IDW and the IDWM methods have similar behavior, the latter experiencing slightly lower variance of MAE but having more "outliers".
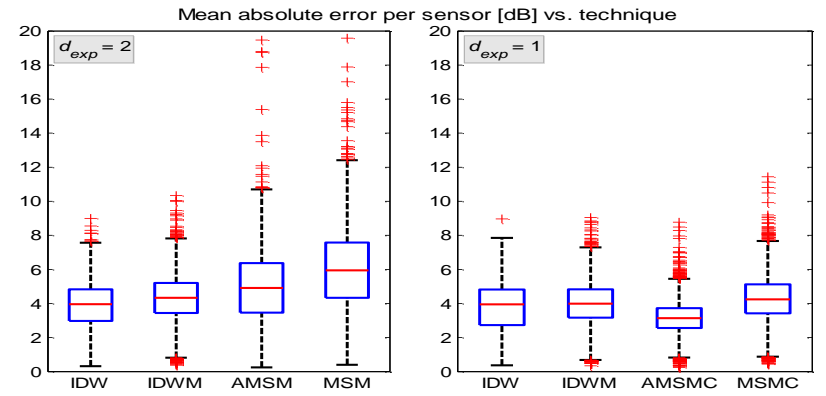

Figure 3. Comparison of IDW based methods in terms of MAE per sensor, impact of distance exponent for interpolation with 9 active sensors.

The following analyses focus only on the interpolation methods having $d_{\exp }$ equal to 1 , due to the better interpolation performances. The focus of the MSM based interpolations remains on the MSMC and the AMSMC approaches, since they provide significantly better performances.

Fig. 4 depicts the box plots presenting the MAE per sensor performances of the IDW interpolation methods of interest with respect to the number of active sensors in the interpolation. As verified by the testbed results, the AMSMC approach (with $d_{\text {exp }}=1$ ) provides the best MAE performances for each case, with the standard deviation of MAE per sensor ranging from $1.04 \mathrm{~dB}$ to $1.56 \mathrm{~dB}$, for nine and five sensors, respectively. However, this interpolation is mostly affected by the decrease of the number of sensors. This is due to the fact that the optimization (adaptation) space of the $N_{q}$ and $N_{w}$ parameters is reduced with the decrease of the number of active sensors. While the classic IDW approaches are more robust to "outliers", the MSM approaches are not.

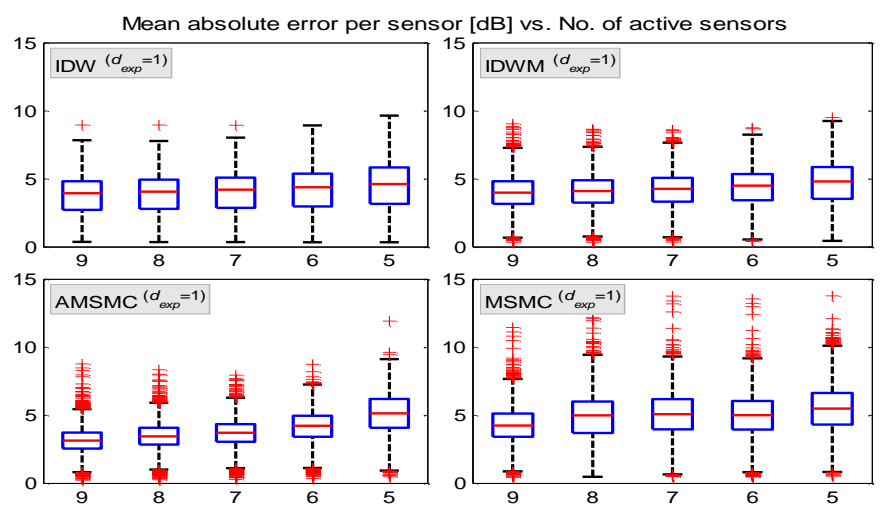

Figure 4. Impact of the number of active sensors to the MAE per sensor for the different IDW based methods.

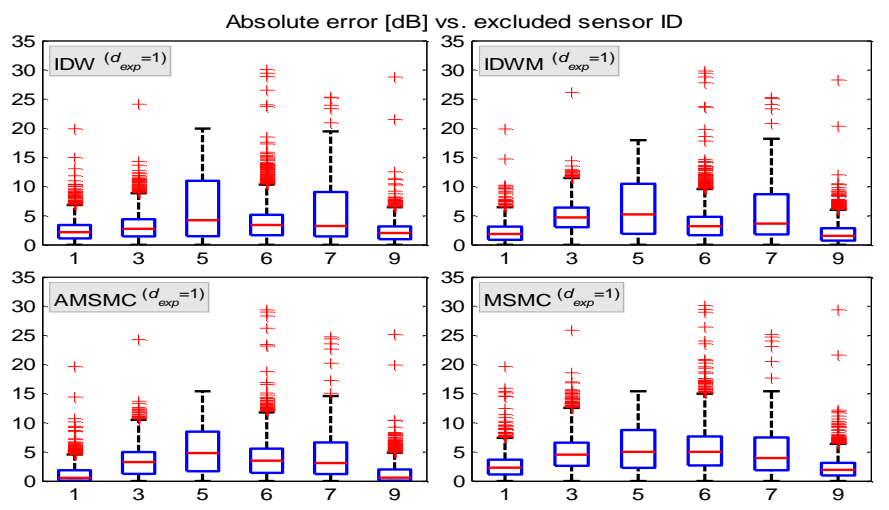

Figure 5. AEs at the excluded sensor ID locations for 9 active sensors.

Fig. 5 presents the dependence of the absolute error on the location of the interpolation point. Namely, the AEs are evaluated at the locations of the excluded sensor, for the case of nine active sensors. The AMSMC interpolation approach again provides the best results for most of the interpolation points (excluded sensors positions). The results show that some of the locations suffer higher interpolation errors, i.e. locations of excluded sensors with IDs 5 and 7 are mostly affected. The standard deviation of the $\mathrm{AE}$ at these sensors positions for the AMSMC approach is $3.74 \mathrm{~dB}$ and $3.44 \mathrm{~dB}$, while for the classic IDW the respective values are $5.03 \mathrm{~dB}$ and $4.53 \mathrm{~dB}$. The error has a negative bias at these locations for all tested IDW methods. This is logical, considering Fig. 2 and the positions of the signal sources - the sensors with IDs 5 and 7 are the closest ones, receiving the highest signal power. When these points are excluded and the dynamic range of the field is high (high power transmissions), the interpolation surface "down-fits" the extremes. Fig. 6 serves to clarify the causes of this effect. It represents the propagation model estimate in the inspected area, obtained with LS fitting of the parameters in the simplified propagation model function [19]. The estimated standard deviation of the shadowing was 
$4.46 \mathrm{~dB}$. Referring to the problem of the high "down-fits" nearby the signal sources, these results prove that these areas are the most critical, since the power drop at distance of $1 \mathrm{~m}$ is around $30 \mathrm{~dB}$ (Fig. 6). The IDW based methods are unable to estimate this extreme in the lack of a nearby observation.

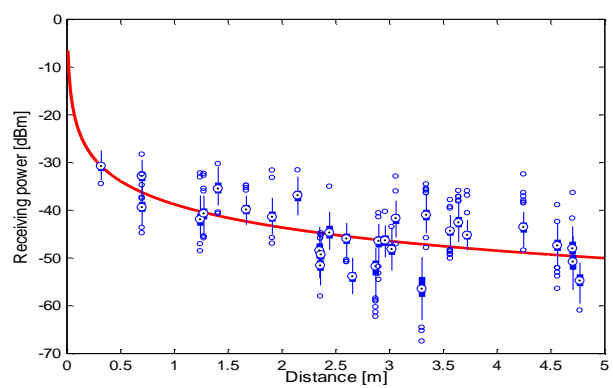

Figure 6. Propagation model estimate in the inspected area.

The results presented in this section serve to prove the applicability of the IDW based methods to the problem of spatial interpolation of RIFs with a low number of observations. Out of all methods, the AMSMC interpolation, with $d_{\text {exp }}$ equal to 1 has proven to offer the lowest interpolation errors. However, the main concern of the IDW based spatial interpolation is the erroneous RIF estimation in nearby transmitter areas. A reasonable approach in these cases would be to use the measurements to perform an initial transmitter location and power estimation. This information can be fed to the IDW based interpolation to reliably synthesize the RIF.

\section{CONCLUSION AND FUTURE WORK}

REMs are practical enablers for cognitive radios and cognitive radio networks. They foster enhanced radio environmental knowledge facilitating adaptive and context aware optimization and resource management decisions. In this paper we have described a practical REM concept elaborating on a general REM architecture and a prototype built on top of a clearly defined REM data model. We have explained RIFs, which form a new key part of the REM data model. REMs and RIFs provide benefit to all stakeholders, i.e. operators (to gain knowledge on their coverage areas), regulators (to track the spectrum usage) and cognitive users (to improve their communication).

The focal point of the paper has been on the reliable estimation of RIFs with small number of measurements, a key challenge in robust construction of REMs. The proposed approach adopts a solution based on IDW spatial interpolation methods, which have proven robustness when dealing with limited number of observations. The standard deviations of the interpolation errors of all inspected methods prove to be in the range of well below ten $\mathrm{dB}$, depending on the measurements number and the employed technique. These interpolation errors as well as the shadowing variance need to be taken into account when calculating the coverage areas, setting up safety margins for cognitive reuse of frequencies, and other related resource management decisions.

Our future work will focus on improvements of the existing IDW techniques, evaluation of additional spatial interpolation methods, and quantifying further the benefits that can be gained by adopting REMs in operational networks.

\section{ACKNOWLEDGMENT}

This work was funded by the EC projects FARAMIR [20] and ACROPOLIS [21]. The authors would like to thank everyone involved. One of us (PM) would like to thank also a partial financial support from DFG through UMIC Research Centre at RWTH Aachen University.

\section{REFERENCES}

[1] J. Mitola, Cognitive Radio: An Integrated Agent Architecture for Software Defined Radio. PhD thesis, KTH (Royal Institute of Technology), 2000.

[2] Y. Zhao et al., "Performance evaluation of radio environment mapenabled cognitive spectrum-sharing networks," IEEE MILCOM 2007, Oct. 2007.

[3] T. Cai et al., "Design of layered radio environment maps for RAN optimization in heterogeneous LTE systems," IEEE PIMRC 2011, Sep. 2011.

[4] J. Riihijärvi et al., "Characterization and modelling of spectrum for dynamic spectrum access with spatial statistics and random fields," IEEE. PIMRC 2008, Sep. 2008.

[5] A. A.-Feki et al., "Informed spectrum usage in cognitive radio networks: Interference cartography," IEEE PIMRC 2008, Sep. 2008.

[6] FARAMIR project deliverable D2.4, "Final System Architecture".

[7] FARAMIR project deliverable D4.1, "Radio Environmental Maps: Information Models and Reference Model". (see ref. 19 for availability)

[8] 3GPP TS 37.320 v10.0.0, "Radio measurement collection for Minimization of Drive Tests (MDT); Overall description; Stage 2".

[9] N. Cressie, “Statistics for spatial data," Wiley Interscience, 1993.

[10] D. Shepard, "A two dimensional interpolation function for irregularly spaced data," in proc. of the 23rd National Conf. of the Association for Computing Machinery, Princeton, NJ, ACM, pp. 517-524, 1968.

[11] R. J. Renka, "Multivariate Interpolation of Large Sets of Scattered Data," ACM Transactions on Mathematical Software, vol. 14, no. 2, pp. 139-148, June, 1988.

[12] N.K. Newlands et al, "Validation and inter-comparison of three methodologies for interpolating daily precipitation and temperature across Canada", EnvironMetrics 22(2), pp. 205-223, 2011.

[13] W. Fa et al., "SAR imaging simulation for an inhomogeneous undulated lunar surface based on triangulated irregular network", Science in China Series F: Information Sciences 52(4), pp. 559-574, 2009.

[14] L. Mitas, H. Mitasova, 'Multivariate approximation by regularized spline with tension", Urbana, National Center for Supercomputing Applications, pp. 1-5, 1997.

[15] V. Atanasovski et al., "Constructing Radio Environment Maps with Heterogeneous Spectrum Sensors", IEEE DySPAN 2011 demonstration, Aachen, Germany, May 2011.

[16] D. Denkovski et al., "Integration of Heterogeneous Spectrum Sensing Devices Towards Accurate REM Construction", IEEE WCNC 2012, Paris, France, April 2012.

[17] S. Pollin et al, "An Integrated Reconfigurable Engine for Multi-purpose sensing up to $6 \mathrm{GHz}$," IEEE DySPAN 2011 demonstration, Aachen, Germany, May 2011.

[18] R. K. Martin, R. W. Thomas, "Algorithms and bounds for estimating location, directionality, and environmental parameters of primary spectrum users, "IEEE Transactions on Wireless Communications 8(11), pp. 5692-5701, 2009.

[19] A. Goldsmith, "Wireless Communications", Cambridge University Press, 2005.

[20] EC FP7 project FARAMIR. http://www.ict-faramir.eu/.

[21] EC FP7 project ACROPOLIS. http://www.ict-acropolis.eu/. 